

Cite this: Phys. Chem. Chem. Phys., 2016, 18, 2292

Received 15th November 2015, Accepted 21st December 2015

DOI: $10.1039 / c 5 c p 06997 a$

www.rsc.org/pccp

\section{The electronic structure of iridium oxide electrodes active in water splitting $\dagger$}

\author{
V. Pfeifer, ${ }^{a b}$ T. E. Jones, ${ }^{* a}$ J. J. Velasco Vélez, ${ }^{a c}$ C. Massué, ${ }^{a c}$ M. T. Greiner, ${ }^{a}$ \\ R. Arrigo, ${ }^{d}$ D. Teschner, ${ }^{a}$ F. Girgsdies, ${ }^{a}$ M. Scherzer, ${ }^{a c} J$. Allan, ${ }^{a}$ M. Hashagen, ${ }^{a}$ \\ G. Weinberg, ${ }^{a}$ S. Piccinin, ${ }^{e}$ M. Hävecker, ${ }^{\text {ac }}$ A. Knop-Gericke ${ }^{a}$ and R. Schlögl ${ }^{a c}$
}

Iridium oxide based electrodes are among the most promising candidates for electrocatalyzing the oxygen evolution reaction, making it imperative to understand their chemical/electronic structure. However, the complexity of iridium oxide's electronic structure makes it particularly difficult to experimentally determine the chemical state of the active surface species. To achieve an accurate understanding of the electronic structure of iridium oxide surfaces, we have combined synchrotron-based X-ray photoemission and absorption spectroscopies with ab initio calculations. Our investigation reveals a pre-edge feature in the $\mathrm{O} \mathrm{K}$-edge of highly catalytically active X-ray amorphous iridium oxides that we have identified as $O 2 p$ hole states forming in conjunction with Ir'I. These electronic defects in the near-surface region of the anionic and cationic framework are likely critical for the enhanced activity of amorphous iridium oxides relative to their crystalline counterparts.

Water splitting presents an attractive solution to store excess energy from intermittent renewable sources in the form of hydrogen. ${ }^{1}$ Iridium oxide is the only known anode electrocatalyst that is both active and stable in the sluggish oxygen evolution reaction (OER) in acidic media, ${ }^{2}$ which has prompted significant research efforts focused on this conducting oxide. Recently, these efforts have revealed that amorphous forms of iridium oxide have higher activities than their rutile-type crystalline counterparts, ${ }^{3,4}$ which we refer to simply as rutile $\mathrm{IrO}_{2}$. An explanation for this increased activity has, however, proven elusive because of the

\footnotetext{
${ }^{a}$ Fritz-Haber-Institut der Max-Planck-Gesellschaft, Faradayweg 4-6, 14195 Berlin, Germany.E-mail: trjones@fhi-berlin.mpg.de

${ }^{b}$ Helmholtz-Zentrum Berlin für Materialien und Energie $\mathrm{GmbH}$, Elektronenspeicherring BESSY II, Albert-Einstein-Str. 15, 12489 Berlin, Germany ${ }^{c}$ Max-Planck-Institut für Chemische Energiekonversion, Stiftstr. 34-36, 45470 Mülheim a. d. Ruhr, Germany

${ }^{d}$ Diamond Light Source Ltd, Harwell Science \& Innovation Campus, Didcot, Oxfordshire OX 11 ODE, UK

${ }^{e}$ Instituto Officina dei Materiali (CNR-IOM), c/o SISSA - Scoula Internazionale Superiore di Studi Avanzati, Via Bonomea 267, 34136 Trieste, Italy

$\dagger$ Electronic supplementary information (ESI) available: Further details on the experiments, measurement results and the DFT calculations are presented. See DOI: $10.1039 / \mathrm{c} 5 \mathrm{cp} 06997 \mathrm{a}$
}

challenges associated with understanding the combined influence of band structure, electron correlation, and spin-orbit coupling on the electronic structure of iridium oxide. ${ }^{5}$ For example, even the Ir 4f spectrum of rutile $\mathrm{IrO}_{2}$ has a line shape that cannot be fit using the standard theoretically derived function, making the origin of this unusual shape a matter of debate. ${ }^{6-8}$ Thus, while X-ray photoemission and absorption spectroscopies (XPS \& XAS) have been used to search for OER relevant surface species in iridium and its oxides, both in situ ${ }^{9-13}$ and $e x$ situ, ${ }^{4,14,15}$ unambiguous speciation has remained challenging. Herein, we combine XPS measurements and theory to uncover the origin of the peculiar Ir $4 \mathrm{f}$ line shape of rutile $\mathrm{IrO}_{2}$. We use this finding along with the Near-Edge X-ray Absorption Fine Structure (NEXAFS) of the $\mathrm{O}$ K-edge to identify the species present in a catalytically more active, amorphous, hydrated $\mathrm{IrO}_{x}$.

All experiments were performed on two commercially available iridium oxide powders. Prior to photoemission experiments, the powders were thoroughly characterized. X-ray diffraction (XRD) confirmed the rutile structure and phase purity of the $\mathrm{IrO}_{2}$ reference material whereas the $\mathrm{IrO}_{x}$ powder was found to be $\mathrm{X}$-ray amorphous with a minor metallic contribution (Fig. S1, ESI $\dagger$ ). Energy-dispersive X-ray spectroscopy (EDX) yielded the expected stoichiometry of the rutile sample $(1: 2 / \operatorname{Ir}: \mathrm{O})$. In contrast, the amorphous powder had a significantly higher oxygen concentration (Table S1, ESI $\dagger$ ) giving rise to a total water loss of $\sim 6 \mathrm{wt} \%$ in thermogravimetric (TG) analysis (Fig. S2, $\mathrm{ESI}+$ ). From temperature-programmed reduction (TPR) (Fig. S3, ESI $\dagger$ ) of rutile $\mathrm{IrO}_{2}$, a formal Ir oxidation state of $4.1 \pm 0.1$ is deduced, demonstrating that iridium and oxygen in rutile $\mathrm{IrO}_{2}$ can be viewed as $\mathrm{Ir}^{\mathrm{IV}}$ and $\mathrm{O}^{\mathrm{II}-}$, despite the distorted octahedral environment around each Ir atom and the covalent character of the Ir-O interaction. ${ }^{16}$ In contrast, the formal oxidation state of oxygen-containing iridium species in the amorphous $\mathrm{IrO}_{x}$ is only found to be $3.6 \pm 0.1$, suggesting that multiple iridium species are present in the amorphous $\mathrm{IrO}_{x}$, some of which are in an oxidation state of less than IV. This result is in line with titration experiments done on hydrated $\mathrm{IrO}_{x}$ colloid catalysts, for which an average oxidation state of 3.2 was found. ${ }^{17}$ 


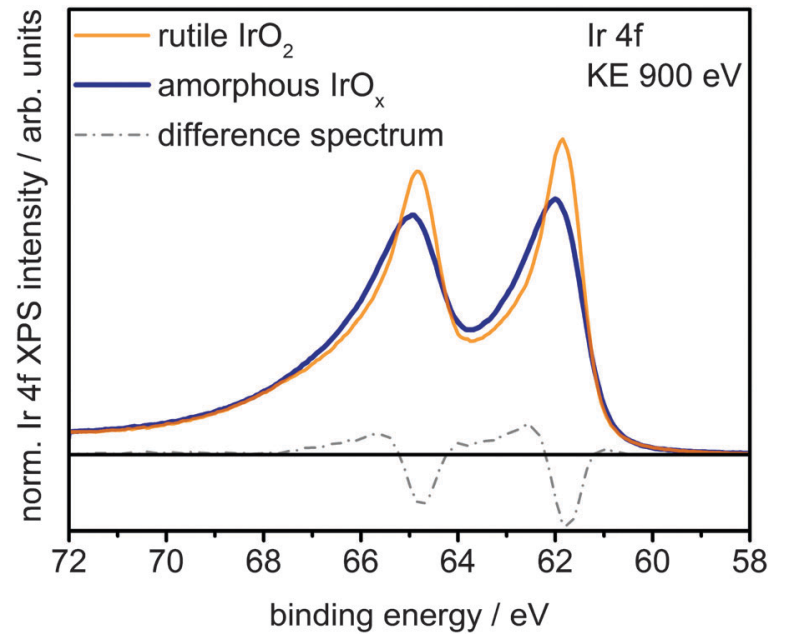

Fig. 1 Ir 4 f spectra and difference spectrum of rutile $\mathrm{IrO}_{2}$ and amorphous $\mathrm{IrO}_{x}$ measured in UHV at a kinetic energy of the photoelectrons of $900 \mathrm{eV}$.

The presence of mixed Ir-valence states has been argued to be essential for the OER on amorphous $\mathrm{IrO}_{x} \cdot{ }^{12,18}$ In support of this view, activity tests of the two powders confirmed the expected higher OER activity of the amorphous $\mathrm{IrO}_{x}$ with respect to rutile $\mathrm{IrO}_{2}$ (Fig. S4, ESI $\dagger$ ). The enhanced activity of amorphous iridium oxides is likely due to surface species that have an intrinsically higher OER activity than those of the rutile $\mathrm{IrO}_{2}{ }^{3}$ To uncover the nature of these species, we turn to XPS and NEXAFS.

Fig. 1 shows a superposition of the measured Ir $4 \mathrm{f}$ spectra of the rutile and the amorphous powders normalized by area. Photoemission of an $\mathrm{Ir} 4 \mathrm{f}_{7 / 2}\left(4 \mathrm{f}_{5 / 2}\right)$ electron in $\mathrm{IrO}_{2}$ gives rise to the intensity at $61.8 \mathrm{eV}(64.8 \mathrm{eV})$ with the anomalous, highly asymmetric line shape typical of rutile $\mathrm{IrO}_{2} \cdot{ }^{19}$ Though the peak positions in the Ir $4 \mathrm{f}$ spectrum of amorphous $\mathrm{IrO}_{x}$ are similar to that of rutile $\mathrm{IrO}_{2}$, the line shape is not, as highlighted by the difference spectrum. The amorphous $\mathrm{IrO}_{x}$ can be seen to have less intensity where the main peaks of rutile $\mathrm{IrO}_{2}$ are located and more intensity around $62.4 \mathrm{eV}$ and $65.4 \mathrm{eV}$. This additional intensity is not related to the metallic component seen in XRD, which would appear at $60.9 \mathrm{eV},{ }^{20}$ suggesting that the metal detected with XRD is covered by a thick oxide layer and does not contribute to the OER activity. The lack of a metallic contribution in XPS makes it tempting to assign the features at $62.4 \mathrm{eV}$ and $65.4 \mathrm{eV}$ to the low oxidation state of iridium seen in TPR. However, the ambiguity in the line shape of $\mathrm{IrO}_{2}$ makes such an assignment questionable without further evidence.

It is well known from many-body theory that, as a conductor, $\mathrm{IrO}_{2}$ has asymmetric core level spectra. ${ }^{19}$ However, the peculiar asymmetry of the Ir $4 \mathrm{f}$ spectrum cannot be fit with only one component when employing the standard Doniach-Šunjic (DS) line shape. ${ }^{6,19}$ Although a variety of hypotheses have been put forward to explain this apparent failure, ${ }^{6-8,21,22}$ it should be noted that the DS function is formally exact only at the edge singularity. The widespread applicability of the DS line shape over a wider energy range is simply a consequence of the weakly structured electron-hole pair excitation spectra common in many metals. ${ }^{23}$ Clearly such weak structure is not required, nor is it always present. While the true line shape can be computed by perturbation theory, ${ }^{19}$ such an approach requires detailed knowledge of the electron-hole pair excitation spectra, hence the atomic structure, and offers little chemical insight. As such, we have chosen to employ a one-electron picture wherein the structured electron-hole pair excitation spectra give rise to "shake-up" satellites, simultaneous core ionization and monopole excitation of a valence electron. While approximate, this approach can be used to understand the experimental spectra and develop robust fit models for complex systems where we lack detailed knowledge of the structure.

To investigate the possibility of shake-up satellites in the Ir uf spectrum of rutile $\mathrm{IrO}_{2}$, we turned to density functional theory (DFT) calculations of the states involved in the shake-up process. The d projected density of states (PDOS(d)) of an Ir atom with an $\mathrm{Ir} 4 \mathrm{f}$ core hole in rutile $\mathrm{IrO}_{2}$ has a strong narrow feature at $1 \mathrm{eV}$ above the Fermi energy $\left(E_{\mathrm{f}}\right)$ (see inset in Fig. 2 and Fig. S9, ESI $\dagger$ ) to which valence electrons can be excited. Because the ground state is metallic and, assuming constant excitation matrix elements, the probability of valence electron excitation scales with the inverse square of the excitation energy $\left(1 / \Delta E^{2}\right)$, the most intense shake-up satellites originate from occupied states near $E_{\mathrm{f}}$. Weaker satellites will originate from states with energies below $E_{\mathrm{f}}$, the most prominent of these will be due to shake-up from localized non-bonding Ir d states at $2 \mathrm{eV}$ binding energy

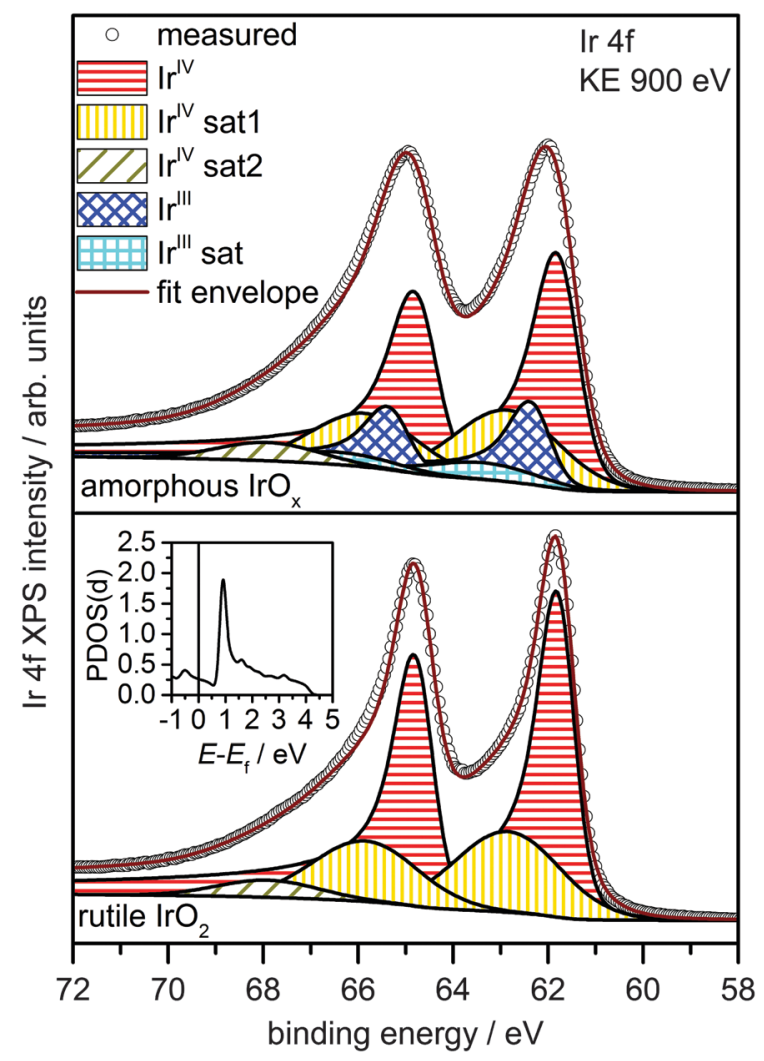

Fig. 2 Theory-based fit models for rutile $\mathrm{IrO}_{2}$ (below) and amorphous $\mathrm{IrO}_{x}$ (above). The inset shows the unoccupied PDOS(d) of rutile $\mathrm{IrO}_{2}$ in presence of an Ir $4 \mathrm{f}$ core hole. The sharp feature $\sim 1 \mathrm{eV}$ above $E_{\mathrm{f}}$ causes the shake-up satellite to appear at $\sim 1 \mathrm{eV}$ above the main line. 
(Fig. S10, ESI $\dagger$ ). Thus, the strongest shake-up satellite will appear at $1 \mathrm{eV}$ higher binding energy than the main line in the Ir $4 \mathrm{f}$ spectrum and a weaker satellite may be seen at $3 \mathrm{eV}$ above the main line.

Using this shake-up picture, the $\mathrm{Ir}^{\mathrm{IV}}$ atoms in the $\mathrm{IrO}_{2}$ rutile structure can be fit as shown in Fig. 2. The fit consists of a DS function with the peak maximum at $61.8 \mathrm{eV}$ and a Gaussian satellite at $1.0 \mathrm{eV}$ higher binding energy. To slightly improve the fit, a small component at $3.1 \mathrm{eV}$ higher binding energy than the Ir $4 \mathrm{f}_{5 / 2}$ line was included to account for shake-up from the localized non-bonding states. The respective satellite corresponding to the Ir $4 \mathrm{f}_{7 / 2}$ line was not included in the fit as it is buried by the main Ir $4 \mathrm{f}_{5 / 2}$ line. It is important to note that, while other authors have also suggested the presence of a $1 \mathrm{eV}$ satellite in the Ir $4 \mathrm{f}$ spectrum due to iridium in an oxidation state greater than $\mathrm{IV},{ }^{22}$ our results show that the main lines and their shake-up satellites all originate from a single type of iridium in the oxidation state IV. All fit parameters can be found in Table S2 (ESI $\dagger$ ).

The presence of principally $\mathrm{Ir}^{\mathrm{IV}}$ and $\mathrm{O}^{\mathrm{II}-}$ in rutile $\mathrm{IrO}_{2}$ is further corroborated by NEXAFS measurements. The strong resonances at $530 \mathrm{eV}$ and $533 \mathrm{eV}$ are in excellent agreement with the computed $\mathrm{O}$ K-edge spectrum of rutile $\mathrm{IrO}_{2}$, where all oxygen is formally $\mathrm{O}^{\mathrm{II}-}$ (Fig. 3). In the $\mathrm{O}$ K-edge spectrum of the amorphous $\mathrm{IrO}_{x}$, however, a new resonance appears at $\sim 529 \mathrm{eV}$, which is also reflected in the $\mathrm{O} 1 \mathrm{~s}$ spectrum (Fig. S5, ESI $\dagger$ ). This type of pre-edge feature is commonly observed in other transition metal oxides with strong covalent interactions. ${ }^{24,25}$ While not an exact comparison, such a pre-edge is perhaps most well-known to emerge when hole doping superconducting cuprates, where it is associated with the formation of $\mathrm{O} 2 \mathrm{p}$ holes. ${ }^{24,25}$

We can computationally test if such formally $\mathrm{O}^{\mathrm{I}-}$ species also lead to a resonance at $\sim 529 \mathrm{eV}$ in iridium oxide by



Fig. 3 Measured $\mathrm{O} \mathrm{K}$-edge spectra of rutile $\mathrm{IrO}_{2}$ and amorphous $\mathrm{IrO}_{x}$ and calculated $\mathrm{O} K$-edges of $\mathrm{O}^{11-}$ and a linear combination of $60 \% \mathrm{O}^{11-}$ and $40 \%$ $\mathrm{O}^{1-}$ species. introducing iridium vacancies to create hole states in $\mathrm{IrO}_{2}$ (Fig. S6 and S8, ESI $\dagger$ ). Doing so, we find that the holes localize on oxygen, which, unlike hole localization on iridium (see $\mathrm{ESI} \dagger$ ), leads to results consistent with experiment. The $\mathrm{O}^{\mathrm{I}-}$ species give rise to a new resonance at $529 \mathrm{eV}$ in the calculated O K-edge, though they lack the resonance at $530 \mathrm{eV}$ seen in the $\mathrm{O}^{\mathrm{II}-}$ spectrum (Fig. S7, ESI $\dagger$ ).

Though this model structure cannot describe all aspects of the amorphous $\mathrm{IrO}_{x}$, by assuming that the electronic structure of the computed $\mathrm{O}^{\mathrm{I}-}$ in $\mathrm{IrO}_{2}$ is similar to that of the analogous coordination defect in the amorphous $\operatorname{IrO}_{x}$, we are able to recover the experimentally observed $\mathrm{O}$ K-edge spectrum. Under this assumption, we can take the model computed spectra as representatives of $\mathrm{O}^{\mathrm{I}-}$ and $\mathrm{O}^{\mathrm{II}-}$, allowing us to conclude that amorphous $\mathrm{IrO}_{x}$ contains both oxygen species within the probing depth of the measurement. This suggestion is supported by the fact that a linear combination of the two types of computed spectra, using 2:3 for the ratio of $\mathrm{O}^{\mathrm{I}-}$ to $\mathrm{O}^{\mathrm{II}-}$, yields good agreement with experiment (Fig. 3). The physisorbed and chemisorbed water content of the $\mathrm{IrO}_{x}$ sample, which was ignored in our computed spectra, leads to a smoothing of the measured $\mathrm{O}$ K-edge spectrum at excitation energies between $537 \mathrm{eV}$ and $545 \mathrm{eV},{ }^{26}$ along with additional intensity at higher binding energy in the O 1s spectrum (see Fig. S5, ESI $\dagger$ ).

The electronic defects in the oxygen seen in NEXAFS also lead to the formation of electronic defects in the iridium framework. Six $\mathrm{O}^{\mathrm{I}-}$ form with each Ir vacancy, leaving two free electrons that reduce neighboring $\operatorname{Ir}^{\mathrm{IV}}$ to $\mathrm{Ir}^{\mathrm{III}}$ to maintain local charge neutrality (Fig. 4). Our calculations reveal that this formally $\mathrm{Ir}^{\mathrm{III}}$ species has an Ir $4 \mathrm{f}_{7 / 2}$ binding energy of $\sim 62.2 \mathrm{eV}$, which is in line with binding energy values measured for $\operatorname{Ir}^{\mathrm{III}}$ in $\mathrm{IrCl}_{3} \cdot{ }^{27}$ As before, assuming that the iridium vacancy modeled in our calculations is representative of coordination defects in the amorphous $\mathrm{IrO}_{x}$, we can use our computed result to conclude that Ir $^{\text {III }}$ can account for the increased intensity at higher binding energy in the Ir $4 \mathrm{f}$ spectrum (see Fig. 1). As with the $\operatorname{Ir}^{\mathrm{IV}}$ species, analysis of the PDOS suggests that a shake-up satellite will appear at $\sim 1.5 \mathrm{eV}$ above the Ir $4 \mathrm{f}$ main line of the $\mathrm{Ir}^{\mathrm{III}}$ species (see Fig. S11, ESI $\dagger$ ).

Based on these theoretical findings, we obtain the fit model shown in Fig. 2 for the amorphous $\operatorname{IrO}_{x}$. The $\mathrm{Ir} 4 \mathrm{f}$ spectrum can be deconvoluted into a contribution of formally $\operatorname{Ir}^{\mathrm{IV}}$ and $\mathrm{Ir}^{\mathrm{III}}$

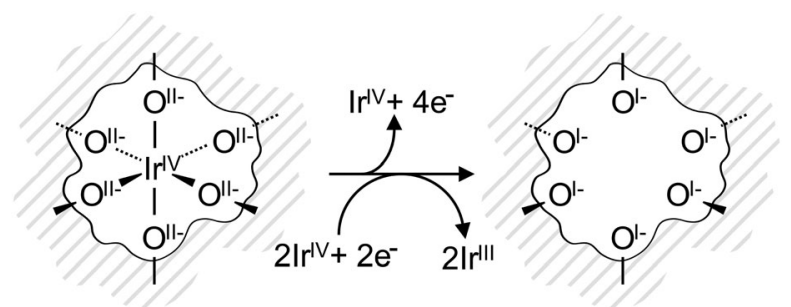

Fig. $4 \quad \mathrm{IrO}_{6}$ octahedron in the near-surface region of the $\mathrm{IrO}_{x}$ framework (grey lines). Initially, Ir and $\mathrm{O}$ are in the formal oxidation states IV and II-, respectively. Upon the creation of an Ir vacancy, surrounding oxygen atoms are oxidized to the formal oxidation state I-. The two additional electrons are distributed among neighboring $\mathrm{Ir}^{\mathrm{IV}}$ reducing them to $\mathrm{Ir}^{\mathrm{III}}$. 
species along with their respective satellites. An increased full width at half maximum of $\mathrm{Ir}^{\mathrm{IV}}$ is used to recover the fact that the local environment around the $\mathrm{Ir}^{\mathrm{IV}}$ atoms in the amorphous structure is expected to be less regular than in the crystalline rutile structure. All fit parameters can be found in Table S3 (ESI $\dagger$ ).

In conclusion, we report on three major findings. First, we have presented theoretical calculations that offer a convincing explanation for the unusual Ir $4 \mathrm{f}$ line shape of phase-pure rutile $\mathrm{IrO}_{2}$. From these results, we developed a fit model, in which a Doniach-Šunjic line shape is combined with Gaussian satellites to account for electron shake-up from $E_{\mathrm{f}}$ into unoccupied states $1 \mathrm{eV}$ above $E_{\mathrm{f}}$. In the second part of the manuscript we have shown that caution is required for the speciation of iridium oxides. For though we observe an increase in intensity at higher binding energy in the Ir $4 \mathrm{f}$ spectrum of the catalytically more active amorphous iridium oxide powder, we see little indication for the presence of Ir in oxidation state V. Both TPR and our calculations suggest that while two Ir components are present in the amorphous iridium oxide, the lower binding energy feature is identified as formally $\mathrm{Ir}^{\mathrm{IV}}$, as in rutile $\mathrm{IrO}_{2}$, whereas the higher binding energy species is attributed to formally $\mathrm{Ir}^{\mathrm{III}}$, which is associated with the formation of $\mathrm{O} 2 \mathrm{p}$ hole states. Finally, these $\mathrm{O} 2 \mathrm{p}$ hole states give rise to a formally $\mathrm{O}^{\mathrm{I}-}$ species. As a chemically electrophilic oxygen, $\mathrm{O}^{\mathrm{I}-}$ is expected to be susceptible to nucleophilic attack during OER, consistent with Fierro et al.' $\mathrm{s}^{28}$ findings that the OER is likely based on the consumption and regeneration of lattice oxygen from the near surface region of the catalyst. Our finding that an electrophilic oxygen can be identified by a resonance at $\sim 529 \mathrm{eV}$ in the O K-edge of the amorphous $\mathrm{IrO}_{x}$, in addition to the $\mathrm{O}^{\mathrm{II}-}$ resonance at $530 \mathrm{eV}$, then allows the study of, not only the active metal centers, but the simultaneous presence of electronic defects in the anionic and cationic framework. These mixed valences of iridium and oxygen likely play a key role in OER catalysis, a finding that might not be restricted to iridium oxides but pertain to OER-active catalysts in general. To aid in our understanding of the intricate roles these species play in reactions, we are investigating their formation and dynamics as a function of applied potential using in situ XPS and NEXAFS.

\section{Experimental and computational methods}

\section{Experimental methods}

The samples investigated were a rutile $\mathrm{IrO}_{2}$ powder (Sigma Aldrich, 99.9\% purity, calcined at $1073 \mathrm{~K}$ in $10^{5} \mathrm{~Pa} \mathrm{O}_{2}$ for $50 \mathrm{~h}$ to ensure phase purity) and an amorphous $\mathrm{IrO}_{x}$ powder (AlfaAesar, 99.99\% purity, as received). Prior to the photoemission measurements, the powders were characterized by XRD, EDX, TG, BET, and TPR. The OER performance was tested in a rotating disc electrode setup. Photoemission measurements were performed with the Near-Ambient-Pressure XPS setup at the ISISS beamline at BESSYII/HZB (Berlin, Germany). Further details can be found in the ESI. $\dagger$

\section{Computational methods}

All Density Functional Theory (DFT) calculations were performed with the Quantum ESPRESSO package ${ }^{29}$ using the Perdew, Burke, and Ernzerhof (PBE) exchange and correlation potential ${ }^{30}$ with spin polarization and scalar relativistic corrections. We employed ultrasoft pseudopotentials with a 50 Ry (500 Ry) kinetic energy (charge density) cutoff and a $k$-point mesh equivalent to $(16 \times 16 \times$ 16) for the crystallographic unit cell along with Marzari-Vanderbilt cold smearing ${ }^{31}$ with a smearing parameter of $0.005 \mathrm{Ry}$. Core level binding energies (BE) were computed using the $\triangle \mathrm{SCF}$ method to accurately recover initial and final state effects. ${ }^{32}$ Oxygen K-edge spectra were computed from Fermi's golden rule using the XSpectra package. ${ }^{3,34}$ Further details are given in the ESI. $\dagger$

\section{Acknowledgements}

The authors gratefully acknowledge BESSYII/HZB for beam time allocation under the proposal \#14201159.

\section{References}

1 J. P. Barton and D. G. Infield, IEEE Trans. Energy Convers., 2004, 19, 441-448.

2 C. C. L. McCrory, S. Jung, J. C. Peters and T. F. Jaramillo, J. Am. Chem. Soc., 2013, 135, 16977-16987.

3 M. Bernicke, E. Ortel, T. Reier, A. Bergmann, J. F. de Araujo, P. Strasser and R. Kraehnert, ChemSusChem, 2015, 8, 1908-1915.

4 T. Reier, D. Teschner, T. Lunkenbein, A. Bergmann, S. Selve, R. Kraehnert, R. Schlögl and P. Strasser, J. Electrochem. Soc., 2014, 161, F876-F882.

5 S. K. Panda, S. Bhowal, A. Delin, O. Eriksson and I. Dasgupta, Phys. Rev. B: Condens. Matter Mater. Phys., 2014, 89, 155102.

6 J. M. Kahk, C. G. Poll, F. E. Oropeza, J. M. Ablett, D. Geolin, J. P. Rueff, S. Agrestini, Y. Utsumi, K. D. Tsuei, Y. F. Liao, F. Borgatti, G. Panaccione, A. Regoutz, R. G. Egdell, B. J. Morgan, D. O. Scanlon and D. J. Payne, Phys. Rev. Lett., 2014, 112, 117601.

7 M. Hara, K. Asami, K. Hashimoto and T. Masumoto, Electrochim. Acta, 1983, 28, 1073-1081.

8 M. Peuckert, Surf. Sci., 1984, 144, 451-464.

9 H. G. S. Casalongue, M. L. Ng, S. Kaya, D. Friebel, H. Ogasawara and A. Nilsson, Angew. Chem., Int. Ed., 2014, 53, 7169-7172.

10 Y. B. Mo, I. C. Stefan, W. B. Cai, J. Dong, P. Carey and D. A. Scherson, J. Phys. Chem. B, 2002, 106, 3681-3686.

11 A. R. Hillman, M. A. Skopek and S. J. Gurman, Phys. Chem. Chem. Phys., 2011, 13, 5252-5263.

12 A. Minguzzi, O. Lugaresi, E. Achilli, C. Locatelli, A. Vertova, P. Ghigna and S. Rondinini, Chem. Sci., 2014, 5, 3591-3597.

13 M. Hüppauff and B. Lengeler, J. Electrochem. Soc., 1993, 140, 598-602.

14 H. N. Nong, L. Gan, E. Willinger, D. Teschner and P. Strasser, Chem. Sci., 2014, 5, 2955-2963.

15 R. Kötz, H. J. Lewerenz, P. Brüesch and S. Stucki, J. Electroanal. Chem. Interfacial Electrochem., 1983, 150, 209-216.

16 Y. Ping, G. Galli and W. A. Goddard, J. Phys. Chem. C, 2015, 119, 11570-11577. 
17 G. S. Nahor, P. Hapiot, P. Neta and A. Harriman, J. Phys. Chem., 1991, 95, 616-621.

18 A. Minguzzi, C. Locatelli, O. Lugaresi, E. Achilli, G. Cappelletti, M. Scavini, M. Coduri, P. Masala, B. Sacchi, A. Vertova, P. Ghigna and S. Rondinini, ACS Catal., 2015, 5, 5104-5115.

19 G. K. Wertheim and H. J. Guggenheim, Phys. Rev. B: Condens. Matter Mater. Phys., 1980, 22, 4680-4683.

$20 \mathrm{~J}$. F. Moulder and J. Chastain, Handbook of X-ray photoelectron spectroscopy a reference book of standard spectra for identification and interpretation of XPS data, Perkin-Elmer, Eden Prairie, Minn., 1992.

21 J. Augustynski, M. Koudelka, J. Sanchez and B. E. Conway, J. Electroanal. Chem., 1984, 160, 233-248.

22 L. Atanasoska, R. Atanasoski and S. Trasatti, Vacuum, 1990, 40, 91-94.

23 G. D. Mahan, Many-particle physics, Springer Science \& Business Media, 2013.

24 N. Nücker, M. Merz, P. Schweiss, E. Pellegrin, S. Schuppler, T. Wolf, V. Chakarian, J. Freeland, Y. U. Idzerda, M. Klaser, G. Müller-Vogt, G. Er, S. Kikkawa and G. Liu, J. Supercond., 1999, 12, 143-146.

25 C. T. Chen, F. Sette, Y. Ma, M. S. Hybertsen, E. B. Stechel, W. M. C. Foulkes, M. Schluter, S. W. Cheong, A. S. Cooper,
L. W. Rupp, B. Batlogg, Y. L. Soo, Z. H. Ming, A. Krol and Y. H. Kao, Phys. Rev. Lett., 1991, 66, 104-107.

26 H. Bluhm, D. F. Ogletree, C. S. Fadley, Z. Hussain and M. Salmeron, J. Phys.: Condens. Matter, 2002, 14, L227.

27 B. Folkesson, Acta Chem. Scand., 1973, 27, 287-302.

28 S. Fierro, T. Nagel, H. Baltruschat and C. Comninellis, Electrochem. Commun., 2007, 9, 1969-1974.

29 P. Giannozzi, S. Baroni, N. Bonini, M. Calandra, R. Car, C. Cavazzoni, D. Ceresoli, G. L. Chiarotti, M. Cococcioni, I. Dabo, A. D. Corso, S. d. Gironcoli, S. Fabris, G. Fratesi, R. Gebauer, U. Gerstmann, C. Gougoussis, A. Kokalj, M. Lazzeri, L. Martin-Samos, N. Marzari, F. Mauri, R. Mazzarello, S. Paolini, A. Pasquarello, L. Paulatto, C. Sbraccia, S. Scandolo, G. Sclauzero, A. P. Seitsonen, A. Smogunov, P. Umari and R. M. Wentzcovitch, J. Phys.: Condens. Matter, 2009, 21, 395502.

30 J. P. Perdew, K. Burke and M. Ernzerhof, Phys. Rev. Lett., 1997, 78, 1396.

31 N. Marzari, D. Vanderbilt, A. De Vita and M. C. Payne, Phys. Rev. Lett., 1999, 82, 3296-3299.

32 E. Pehlke and M. Scheffler, Phys. Rev. Lett., 1993, 71, 2338-2341. 33 C. Gougoussis, M. Calandra, A. P. Seitsonen and F. Mauri, Phys. Rev. B: Condens. Matter Mater. Phys., 2009, 80, 075102. 34 M. Taillefumier, D. Cabaret, A. M. Flank and F. Mauri, Phys. Rev. B: Condens. Matter Mater. Phys., 2002, 66, 195107. 